\title{
Equivalent Modeling Method for Regional Decentralized Photovoltaic Clusters Based on Cluster Analysis
}

\author{
Hongbin $\mathrm{Wu}$, Zhongqian Liu, Yu Chen, Bin Xu, and Xianjun Qi
}

\begin{abstract}
A large number of photovoltaic (PV) systems are decentralized and connected to distribution networks, leading to challenges in model simulation. This paper presents an equivalent modeling method for regional decentralized PV clusters based on cluster analysis. The proposed method is based on the grouping principle that PV systems have similar dynamic response characteristics, and grouping results are obtained using a fuzzy $\mathrm{C}$-mean (FCM) clustering algorithm. Based on the clustering evaluation index, the optimal grouping number can then be determined and PV systems in the same group are combined into an equivalent PV system. The method to acquire equivalent parameters for PV arrays, transformers, and lines is also presented. A multi-machine equivalent model of regional decentralized PV clusters is established, and the precision and efficiency of the proposed method is demonstrated using an actual distribution network.
\end{abstract}

Index Terms-Distribution network, equivalent modeling, fuzzy C-mean clustering, photovoltaic cluster, regional decentralization.

\section{INTRODUCTION}

$\mathrm{S}$ OLAR energy is a clean, renewable energy that has undergone rapid development and large-scale application, with photovoltaic (PV) grid-connected systems being the most important solar energy application [1]-[3]. In China, with the implementation of $\mathrm{PV}$ power generation poverty reduction policies, many PV power stations are decentralized and connected to distribution networks, forming regional decentralized PV clusters [4]. This increases the complexity of the distribution network model and leads to challenges such as long simulation times, huge memory usages, and difficulty updating models. Therefore, it is necessary to establish a simplified equivalent model of regional decentralized PV clusters.

In recent years, equivalent modeling of PV system research has concentrated primarily on regional centralized PV systems or large-scale PV power stations. In [5], the authors propose an online clustering modeling method for large-scale PV power

Manuscript received February 7, 2018. This work was supported by the National Key R\&D Program of China under Grant 2016YFB0900400.

$\mathrm{H}$. Wu, Z. Liu and X. Qi are with the School of Electrical Engineering and Automation, Hefei University of Technology, No. 193, Tunxi Rd., Hefei 230009, China (e-mail: hfwuhongbin@163.com; 15695659223@163.com; qxj_216@163.com).

Y. Chen is with the State Grid Anhui Economic Research Institute, No. 1599, Huaining Rd., Hefei, China (e-mail: cy39fly@126.com).

B. Xu is with the State Grid Anhui Electric Power Research Institute, No. 299, Ziyun Rd., Hefei, China (e-mail: xubin1980@sina.com).

Digital Object Identifier 10.24295/CPSSTPEA.2018.00014 plants, using the control parameters of inverter as the clustering index. In [6], two equivalent modeling methods for PV system are proposed for partially shielded and unshielded PV cells. To study the transient characteristics of large-scale PV power stations and avoid constructing detailed models for every type of inverter, Mukherjee et al. [7] analyzed the effectiveness of the model reference adaptive control (MRAC) approach for modeling a grid connected inverter system. In [8], the equivalent dynamic model of an actual PV power station with virtual synchronous characteristics was studied, and a method was proposed to obtain the dynamic equivalent model of a group of PV converters based on the synchronous power controller.

The study of equivalent modeling of regional decentralized PV system is in its infancy. In the coherent-based equivalent method of large-scale power grids, the coherent generator group is equivalent to corresponding aggregates, and only key transmission lines and nodes are retained. Therefore, the power system scale can be reduced and the main dynamic characteristics can be preserved [9]-[11]. However, the PV power generation is different from conventional power generation, so the criterion for PV system coherency must be defined to benefit from a coherency-based equivalent method. PV systems with similar dynamic responses under the same disturbance are considered coherent, and they should be combined into an equivalent PV system to ensure the dynamic characteristics of the original system remain unchanged.

This paper proposes an equivalent modeling method for regional decentralized PV clusters. The clustering index is selected from the response curves of $\mathrm{PV}$ systems during a disturbance. Then, a fuzzy C-mean (FCM) clustering algorithm is used to obtain dynamic grouping results, and PV systems in the same group are combined into an equivalent PV system, establishing a multi-machine equivalent model of regional decentralized PV clusters. Finally, simulation analysis is performed using an actual distribution network in Anhui province.

\section{Clustering InDEX}

\section{A. Model of Regional Decentralized PV Cluster}

A PV cluster is a collection of PV systems that are near each other with only small differences in solar radiation, and the same point of interconnection (PCC). Taking the city of Jinzhai, Anhui province as an example, a total of 295 small village PV systems have been built and connected to a distribution net- 


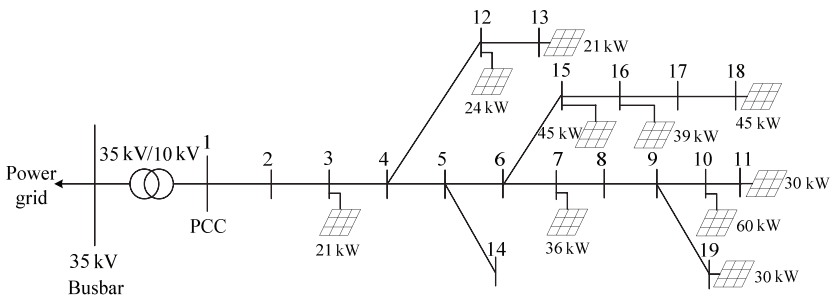

Fig. 1. Regional decentralized PV clusters.

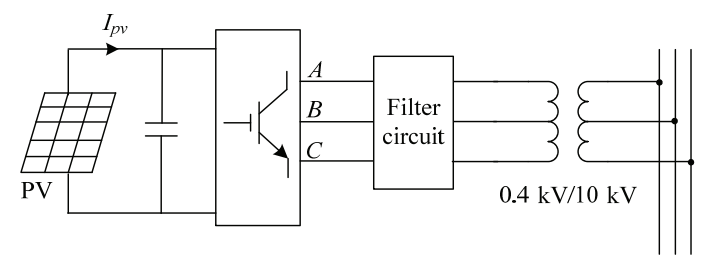

Fig. 2. The structure of a single PV system.

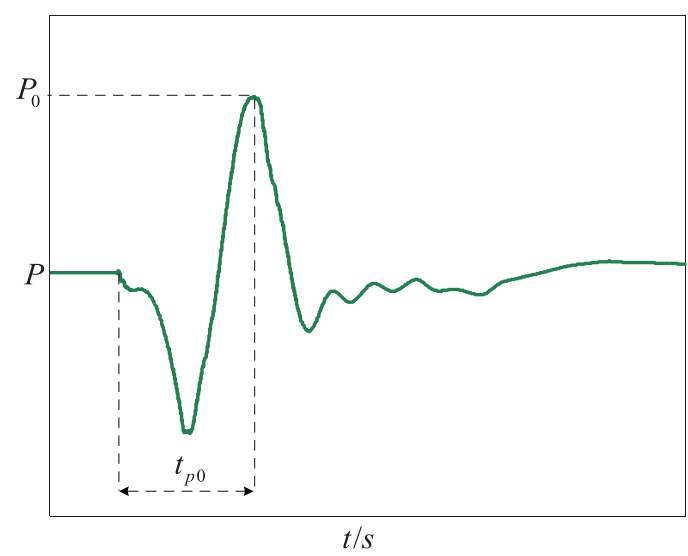

Fig. 3. Sketch of active power disturbance curve extraction.

work, forming several regional decentralized PV clusters. A typical PV cluster under a $35 \mathrm{kV}$ substation is shown in Fig. 1, there are $10 \mathrm{PV}$ systems in all, which is called the detailed model.

The common model of a single PV system is shown in Fig. 2, which contains a PV array, a grid-connected inverter, a filter, and other devices. The specific characteristic equation can be referred to in [12], but is not described in detail in this article.

\section{B. Clustering Index of PV System}

The basic idea of a regional decentralized PV cluster is to combine PV systems that have similar dynamic responses into an equivalent PV system. However, dynamic features of PV systems are influenced by many factors, including inverter controller parameters, the location and capacity of PV systems, and other parameters. Therefore, it is difficult to propose a reasonable clustering index from inherent parameters of a system model [13]. In this paper, the feature points extracted from the response curve of a PV system are taken as the clustering index. The active power response curve of a PV system under typical disturbance is shown in Fig. 3.

In Fig. $3, P_{0}$ is the first peak and $t_{p 0}$ is the time from the start of the dynamic process to the first peak. For the reactive power response curve, extraction of feature points is the same as for active power, with the two feature points being recorded as $Q_{0}$ and $t_{q 0}$, respectively.

Considering that under a three-phase short-circuit disturbance, the dynamic response of a PV system is generally the most obvious, when a three-phase short-circuit fault occurs at the PCC point, the vector $\left[P_{0}, t_{p 0}, Q_{0}, t_{q 0}\right]$ of each PV system is taken as the clustering index. Note that parameters must be normalized due to differences in dimensions and orders of magnitude of parameters.

\section{Multi-Machine Equivalence of Regional Decentralized PV Clusters}

\section{A. Clustering Method of PV Systems Based on the FCM Al- gorithm}

The clustering algorithm is an iterative optimization process, where the similarity between the clustering index which are in the same group is greater, and the similarity between clustering index of different groups is smaller[14]-[18]. On the basis of general clustering, the concept of fuzzy clustering is proposed. Through the FCM algorithm, $n$ clustering indexes $x_{j}(j=1,2, \ldots, n)$ that corresponding to $n \mathrm{PV}$ systems are divided into $c$ groups, it is determined which group each clustering index belongs to by the membership between $[0,1]$, and the clustering centers of each group are calculated $v_{i}(i=1,2, \ldots, c)$ to get the minimal value of the objective function. The objective function is as follows:

$$
J_{m}(U, c)=\sum_{i=1}^{c} \sum_{j=1}^{n} \mu_{i j}^{m}\left\|v_{i}-x_{j}\right\|_{2}
$$

where $\left\|v_{i}-x_{j}\right\|_{2}$ is the Euclidean distance between the $i^{\text {th }}$ cluster center $v_{i}$ and the clustering index of the $j^{\text {th }} \mathrm{PV}$ system $x_{j}, m$ is the weighting exponent, $m \in[1, \infty)$, usually $m$ is $2, c \in[2, \sqrt{n}], u_{i j}$ is the membership value that the $j^{\text {th }}$ PV system belongs to the $i^{\text {th }}$ cluster center, which satisfies normalization:

$$
\text { s.t. } \begin{cases}\sum_{i=1}^{c} \mu_{i j}=1, & 1 \leq j \leq n \\ \mu_{i j} \in[0,1], \quad 1 \leq j \leq n, & 1 \leq i \leq c \\ n>\sum_{j=1}^{n} \mu_{i j}>0, & 1 \leq i \leq c\end{cases}
$$

In order for (1) to achieve the minimum value, the following functions are constructed:

$$
\bar{J}_{m}(U, c, \lambda)=J_{m}(U, c)+\sum_{j=1}^{n} \lambda_{j}\left(\sum_{i=1}^{c} u_{i j}-1\right)
$$

where $\lambda_{j}$ is the Lagrange multiplier of (2). By taking the derivative of all input parameters, we can get the necessary condition for (1) to get the minimum value, which is as follows: 


$$
\begin{gathered}
v_{i}=\sum_{j=1}^{n} \mu_{i j}{ }^{m} x_{j} / \sum_{j=1}^{n} \mu_{i j}{ }^{m} \\
\mu_{i j}=\frac{1}{\sum_{k=1}^{c}\left(\frac{\left\|x_{j}-v_{i}\right\|_{2}}{\left\|x_{j}-v_{k}\right\|_{2}}\right)^{\frac{2}{m-1}}}
\end{gathered}
$$

Thus, the output of the FCM algorithm includes two parts: the first part is $c$ clustering centers, and each clustering center represents the average characteristics of the clustering index of the PV systems, the second part is a $c \times n$-order membership matrix, which represents the membership that each PV system belongs to each group. Generally, the rule of maximum membership determines which group the PV systems belong to.

To evaluate the clustering effect and determine the optimal grouping number, two evaluation indices, the partition coefficient $(P C)$ and the classification entropy $(C E)$, are defined as follows:

$$
\begin{gathered}
P C=\frac{1}{n} \sum_{i=1}^{c} \sum_{j=1}^{n}\left(\mu_{i j}\right)^{2} \\
C E=-\frac{1}{n} \sum_{i=1}^{c} \sum_{j=1}^{n} \mu_{i j} \log \left(\mu_{i j}\right)
\end{gathered}
$$

where PC is used to evaluate the degree of separation of different $\mathrm{PV}$ groups, the larger the better, and $\mathrm{CE}$ is used to evaluate the degree of fuzziness of different PV groups, the smaller the better.

\section{B. Multi-Machine Equivalent Model}

Using the FCM algorithm to group PV systems in Fig. 1, the detailed steps are shown as follows:

Step1: The clustering index $\left[P_{0}, t_{p 0}, Q_{0}, t_{q 0}\right]$ are constructed for all PV systems.

Step2: A membership matrix is generated randomly, which satisfy (2).

Step3: Updating the clustering centers through the membership matrix according to (4).

Step 4: Updating the membership matrix through the clustering centers according to (5).

Step5: Repeating steps 3 and 4 until the change of the value of the objective function calculated by (1) is less than the permissible value.

Step 6: Calculating the clustering evaluation indices and according to (6) and (7).

Step 7: Changing the number of groups $c$, and come back to step 2 to group the PV systems again. The best number of groups is determined by comparing the values of $P C$ and $C E$ under different number of groups, and the final grouping results of PV system are determined by the membership matrix at this time.

The $n$ PV systems are divided into $c$ groups through the above steps, then PV systems that are in the same group are merged into an equivalent PV system, all equivalent PV systems are then connected to the PCC. The equivalent model is shown in Fig. 4.

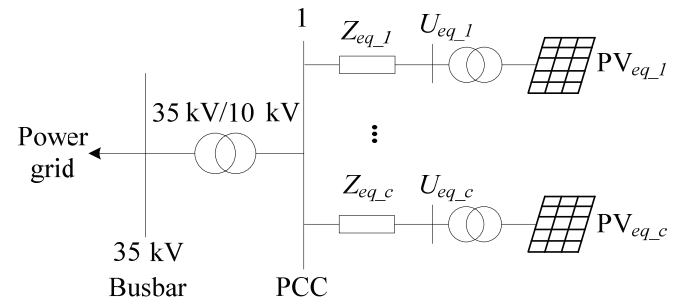

Fig. 4. Equivalent model of regional decentralized PV cluster.

The equivalent model of regional decentralized PV clusters is required to have the same external characteristics as the detailed model. That is to say, the two models should have similar dynamic response curves at the PCC point, including power, frequency, current, voltage, and so on.

\section{Equivalent Parameters Calculation}

In Fig. 4, taking the $x^{\text {th }}(1 \leq x \leq c)$ equivalent PV system as an example, and supposing that the $x^{\text {th }}$ equivalent PV system is merged by $M$ PV systems, the calculation method of the equivalent parameters is as follows.

\section{A. Equivalence of PV Array}

In the actual operation, it is difficult to ensure the power generation of PV systems due to partial shading and passing clouds, at this point, we can adopt parameter identification method to get the equivalent number of PV modules under ideal condition according to reference [19], thus the actual power generation of each PV array can be obtained, denoted as $P_{k}(k=1,2, \ldots, M)$. The power generation of the $x^{\text {th }}$ equivalent $\mathrm{PV}$ array is as follows:

$$
P_{e q_{-} x}=\sum_{k=1}^{M} P_{k}
$$

\section{B. Equivalence of Line Parameters}

Before equivalence, the voltage difference between the $M \mathrm{PV}$ systems and the PCC can be obtained by the power flow calculation, which is denoted as $\Delta U_{k}(k=1,2, \ldots, M)$. Then we can calculate the weighted average voltage difference of the $M \mathrm{PV}$ systems (the power of a PV system is used for weighting) as:

$$
\Delta \bar{U}_{x}=\sum_{k=1}^{M}\left(\Delta U_{k} P_{k}\right) / \sum_{k=1}^{M} P_{k}
$$

After equivalence, according to Fig. 4, the voltage difference between the $x^{\text {th }}$ equivalent PV system and the PCC is calculated as follows:

$$
\Delta \bar{U}_{e q_{-} x}=\frac{Z_{\text {eq_}} P_{\text {eq_}} x}{\sqrt{3} U_{P C C}}
$$

where $U_{P C C}$ is the voltage of at the PCC point .

Solving the equation $\Delta \bar{U}_{x}=\Delta \bar{U}_{e q_{-} x}$, the impedance of the equivalent line is as follows: 


$$
Z_{e q_{-} x}=\frac{\sqrt{3} U_{p c c} \sum_{k=1}^{M}\left(\Delta U_{k} P_{k}\right)}{\left(\sum_{k=1}^{M} P_{k}\right)^{2}}
$$

The above is the equivalent method of line impedance. Because the length of the line is short and the voltage is low, the influence of line capacitance can be ignored.

\section{Equivalence of Transformer Parameters}

According to Fig. 2, the equivalent impedance circuit of a single PV system can be simplified as shown in Fig. 5. where $Z_{t r k}$ is the impedance of the $k^{\text {th }} \mathrm{PV}$ transformer. Therefore, before equivalence, the total loss of the $M$ transformers can be calculated by:

$$
S_{x}=\sum_{k=1}^{M} I_{k}^{2} Z_{t r-k}
$$

After equivalence, the loss of the transformer in the $x^{\text {th }}$ equivalent PV system is:

$$
S_{e q_{-} x}=I_{e q_{-}}{ }^{2} Z_{t r-e q \_} x
$$

At the same voltage level, it is thought that $I \propto P$. Therefore, by solving the equation $S_{x}=S_{\text {eq_ }}$, the impedance of the $x^{\text {th }}$ equivalent transformer can be obtained:

$$
Z_{t r_{-} e q_{-} x}=\frac{\sum_{k=1}^{M}\left(P_{k}^{2} Z_{t r_{-} k}\right)}{\left(\sum_{k=1}^{M} P_{k}\right)^{2}}
$$

Due to the low voltage level, the loss of the transformer excitation branch is small and can be ignored.

\section{Equivalence of Inverter Parameters}

The equivalent PI controller parameter of grid-connected inverters can be calculated using the capacity weighted method [20] as follows:

$$
X_{\text {eq_ } x}=\sum_{k=1}^{M}\left(X_{k} P_{k}\right) / \sum_{k=1}^{M} P_{k} \quad X=k_{p u}, k_{i u}, k_{p i}, k_{i i}
$$

where $k_{p u}$ and $k_{i u}$ are the proportional and integral coefficients of the voltage outer loop, respectively, and $k_{p i}$ and $k_{i i}$ are the proportional and integral coefficients of the current inner loop, respectively.

To ensure the filtering effect of equivalent inverters, and that reactive power consumption remains unchanged before and after the equivalence, the equivalent filter inductance can be acquired by:

$$
L_{e q \_}=\sum_{k=1}^{M} L_{k} / M^{2}
$$

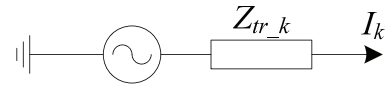

Fig. 5. Equivalent impedance circuit of a single PV system.

\section{E. Error Analysis of Equivalent Model}

In order to analyze the error of equivalent model proposed in this paper, the simulation results of the detailed model of PV cluster are taken as the reference, the error evaluation index of equivalent model is calculated as follows:

$$
E=\int_{t_{1}}^{t_{2}}\left|\frac{Y_{d}-Y_{e}}{Y_{d}}\right| d t
$$

where $t_{1}$ and $t_{2}$ are the start time and end time of simulation, respectively, $Y_{d}$ and $Y_{e}$ are the electrical quantity of the detailed model and the equivalent model at PCC, respectively, the electrical quantity here can be active power, reactive power, frequency and so on.

\section{NumericAl EXAMPLES}

\section{A. Example of the System}

The simulation system is the same as shown in Fig. 1. A typical double-loop controller is used as the inverter controller model, which contains a power outer loop and a current inner loop. The reference value of reactive power is set to zero, making the power factor unity. The main parameters of the system are shown in TABLE I and TABLE II. The line model is LGJ-35.

\section{B. Clustering Result Analysis}

The FCM algorithm is used to group the above regional decentralized PV cluster, when the grouping number is different, the evaluation indices of PC and CE are shown in Fig. 6.

As can be seen in Fig. 6, when the regional decentralized PV cluster is divided into two groups, the evaluation index PC is at a maximum and $\mathrm{CE}$ is at a minimum. Therefore, the optimal grouping number is $c=2$. Results are shown in TABLE III.

The equivalent model of the regional decentralized PV cluster was calculated based on the method presented in Section III and is given in TABLE III.

\section{Equivalence Effect Verification}

In order to verify the effect of the equivalent model, the following four cases are simulated:

\section{1) Three-Phase Short Circuit Fault}

This simulation will compare the proposed equivalent method with the traditional single-machine equivalent method to verify the method in this paper. A three-phase short circuit fault occurs at the PCC point at $0.3 \mathrm{~s}$ and the fault clears at $0.38 \mathrm{~s}$. The response curves of the detailed model, the proposed equivalent model, and the traditional single-machine equivalent model at PCC are shown in Fig. 7, the error calculation results are shown 
TABLE I

Line PARAMETERS

\begin{tabular}{cccc}
\hline \hline Line & Length $(\mathrm{m})$ & Line & Length $(\mathrm{m})$ \\
\hline $1-2$ & 1839 & $10-11$ & 435 \\
$2-3$ & 1266 & $4-12$ & 241 \\
$3-4$ & 1679 & $12-13$ & 1180 \\
$4-5$ & 583 & $5-14$ & 146 \\
$5-6$ & 115 & $6-15$ & 1610 \\
$6-7$ & 386 & $15-16$ & 1528 \\
$7-8$ & 1298 & $16-17$ & 985 \\
$8-9$ & 90 & $17-18$ & 708 \\
$9-10$ & 172 & $9-19$ & 2319 \\
\hline \hline
\end{tabular}

TABLE II

PV NODE PARAMETERS

\begin{tabular}{cccccccc}
\hline \hline \multirow{2}{*}{$\begin{array}{c}\text { Node } \\
\text { Number }\end{array}$} & Power & \multicolumn{6}{c}{ Inverter Parameters } \\
& $(\mathrm{kW})$ & $k_{p u}$ & $k_{i u}$ & $k_{p i}$ & $k_{i i}$ & $L_{k}(\mathrm{mH})$ & Transformer \\
Type \\
\hline 3 & 21 & 0.51 & 0.86 & 150 & 10 & 0.125 & $\mathrm{~S} 11-100 / 10$ \\
7 & 36 & 0.80 & 2.55 & 100 & 5 & 0.125 & $\mathrm{~S} 11-125 / 10$ \\
10 & 60 & 0.62 & 1.23 & 400 & 1 & 0.125 & $\mathrm{~S} 11-\mathrm{M}-200 / 10$ \\
11 & 30 & 0.45 & 3.24 & 200 & 8 & 0.125 & $\mathrm{~S} 11-\mathrm{M}-200 / 10$ \\
12 & 24 & 0.75 & 1.05 & 300 & 2 & 0.125 & $\mathrm{~S} 11-160 / 10$ \\
13 & 21 & 0.50 & 1.56 & 350 & 6 & 0.125 & $\mathrm{~S} 11-100 / 10$ \\
15 & 45 & 0.42 & 0.65 & 250 & 1 & 0.125 & $\mathrm{~S} 9-100 / 10$ \\
16 & 39 & 0.35 & 2.20 & 100 & 10 & 0.125 & $\mathrm{~S} 11-\mathrm{M}-100 / 10$ \\
18 & 45 & 0.62 & 1.64 & 400 & 4 & 0.125 & $\mathrm{~S} 9-100 / 10$ \\
19 & 30 & 0.80 & 2.76 & 150 & 5 & 0.125 & $\mathrm{~S} 11-\mathrm{M}-100 / 10$ \\
\hline \hline
\end{tabular}

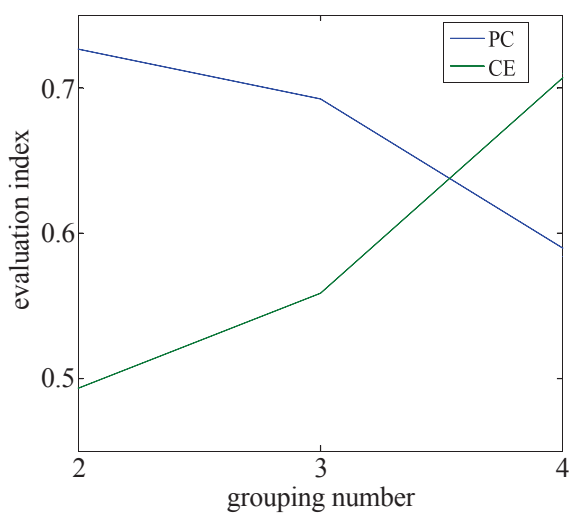

Fig. 6. Evaluation index of the clustering effect.

in TABLE IV, the actual time spent in the simulation process of the three models are shown in TABLE V.

As shown in Fig. 7 and TABLE IV, the equivalent model in this paper has higher precision than the traditional single-machine model. TABLE V shows that the simulation time of the equivalent model in this paper is much lower than that of the detailed model, and is close to that of the traditional single-machine equivalent model. Therefore, the proposed equivalent model in this paper balances precision and simplification. However, this method also has some limitations, when the structure of distribution network or the capacity of PV systems changes,
TABLE III

Best Grouping Results

\begin{tabular}{cc}
\hline & PV System Node Number \\
\hline Group 1 & $3,7,11,12,13,19$ \\
Group 2 & $10,15,16,18$ \\
\hline \hline
\end{tabular}

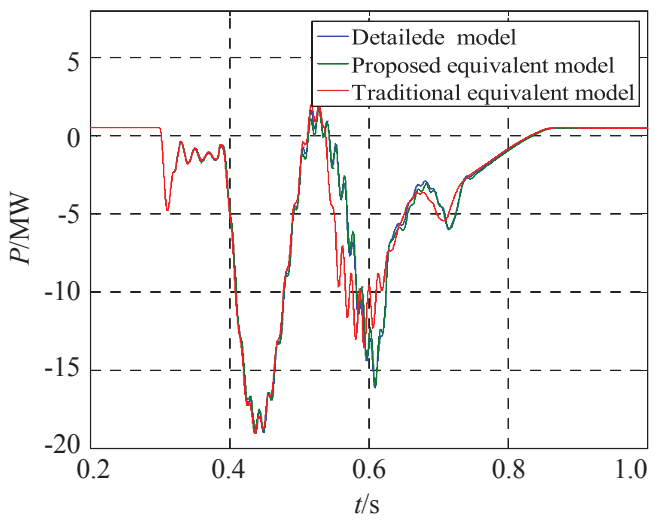

(a) Active power response curve.

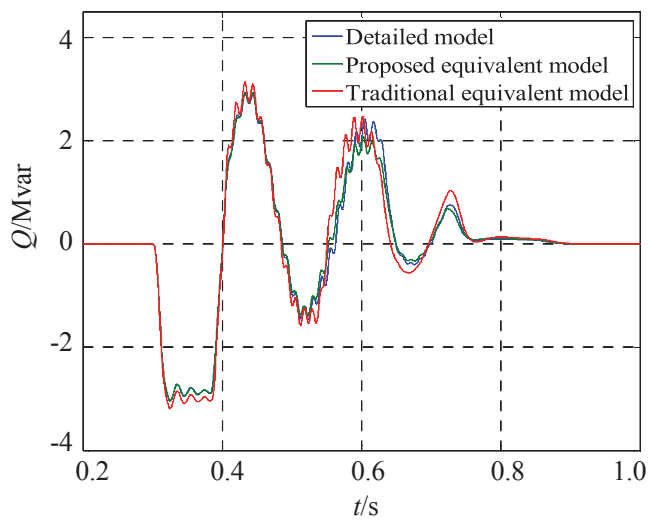

(b) Reactive power response curve.

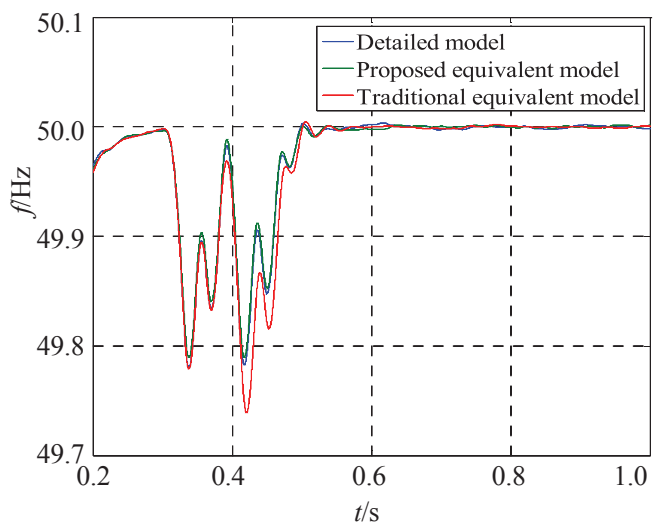

(c) Frequency response curve.

Fig. 7. Response curves for three-phase short circuit.

the power response curve under typical disturbance shown in Fig. 3 will also change, which leads to the change of clustering index, therefore we need to rebuild the clustering index for each PV system, it will increase much calculation burden. 
TABLE IV

Error Calculation Results

\begin{tabular}{ccc}
\hline \hline & $\begin{array}{c}\text { Proposed } \\
\text { equivalent model }\end{array}$ & $\begin{array}{c}\text { Traditional } \\
\text { equivalent model }\end{array}$ \\
\hline Active power error & 0.0041 & 0.0115 \\
Reactive power error & 0.0095 & 0.0340 \\
Frequency error & 0.0018 & 0.0044 \\
\hline \hline
\end{tabular}

TABLE V

Model Simulation Time

\begin{tabular}{cccc}
\hline \hline & Detailed model & $\begin{array}{c}\text { Proposed } \\
\text { equivalent model }\end{array}$ & $\begin{array}{c}\text { Traditional } \\
\text { equivalent model }\end{array}$ \\
\hline Time spent & $25 \mathrm{~s}$ & $7 \mathrm{~s}$ & $5 \mathrm{~s}$ \\
\hline \hline
\end{tabular}

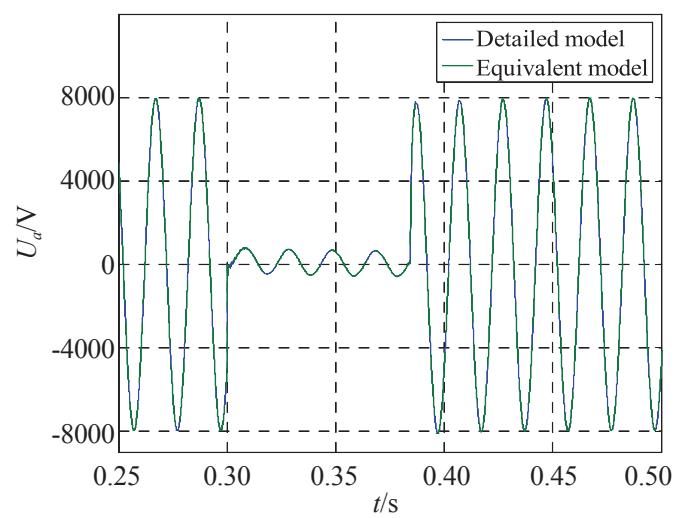

(a) Phase A voltage.

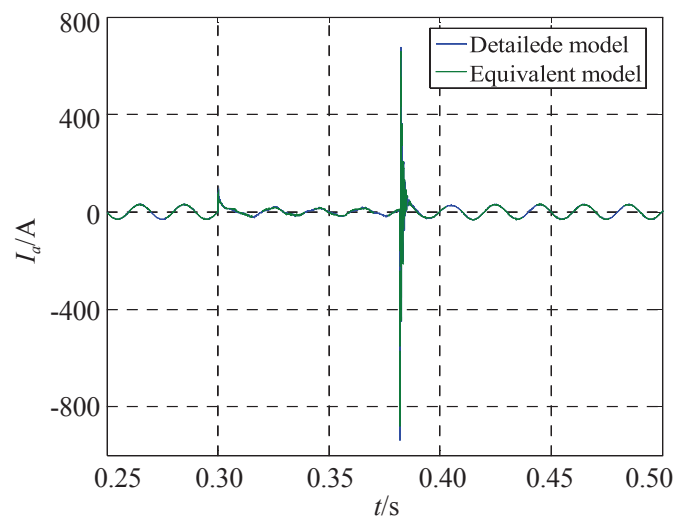

(b) Phase A current.

Fig. 8. Voltage and current response curves for single-phase short circuit.

As a final note, the equivalent model in this paper is a two-machine equivalent model based on TABLE III, but it does not mean that this equivalent model has the highest precision. In general, the more grouping number, the higher precision of the established equivalent model, but the model will be more complex correspondingly. In this paper, the best grouping number is chosen by the calculation of PC and CE, so the established equivalent model can best balance precision and simplification in theory.

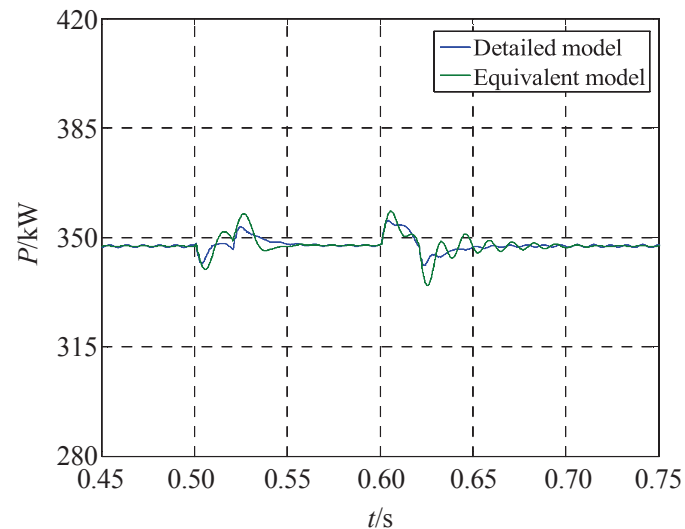

(a) Active power response curve.

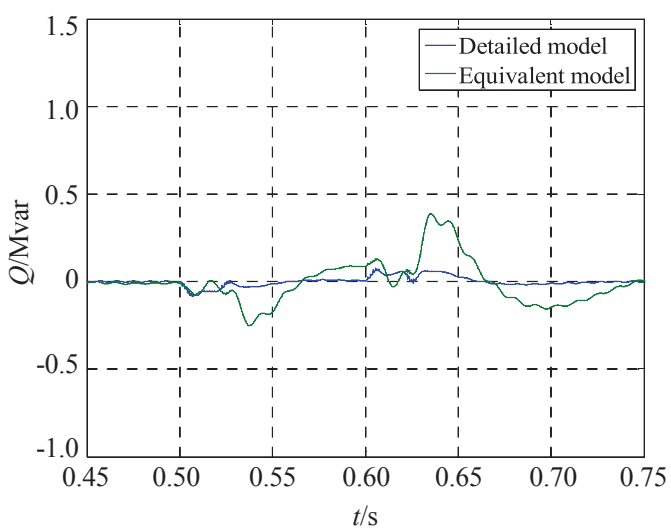

(b) Reactive power response curve.

Fig. 9. Response curves for voltage disturbance from power grid.

\section{2) Single-Phase Short Circuit Fault}

The clustering index of the PV system is constructed under the condition of a three-phase short circuit fault as shown in section II. To verify the application of the equivalent model to another fault situation, a single-phase ground fault occurs at PCC point at $0.3 \mathrm{~s}$ and the fault clears at $0.38 \mathrm{~s}$. Voltage and current curves of the detailed model and the equivalent model at the PCC point are shown in Fig. 8.

Fig. 8 shows that the external characteristics of the equivalent model are in agreement with that of the detailed model. Therefore, the clustering index presented in this paper is reasonable, and a PV system with similar dynamic characteristics can be divided into the same group by the clustering algorithm.

\section{3) Voltage Disturbance From Power Grid}

Three-phase short circuit fault and single-phase short circuit fault are both large disturbance, in order to verify the accuracy of the equivalent model under small disturbance, small disturbance of power grid voltage is set in this simulation: the power grid voltage change from 1.0 p.u to 0.9 p.u at $0.5 \mathrm{~s}$, and the voltage is restored to $1.0 \mathrm{p} . \mathrm{u}$ at $0.6 \mathrm{~s}$. The active and reactive power curves of the detailed model and the equivalent model at PCC are shown in Fig. 9.

As seen in Fig. 9, the response curve of equivalent model is still agreement with the detailed mode under small voltage disturbance from power grid, this comparison shows the effective- 


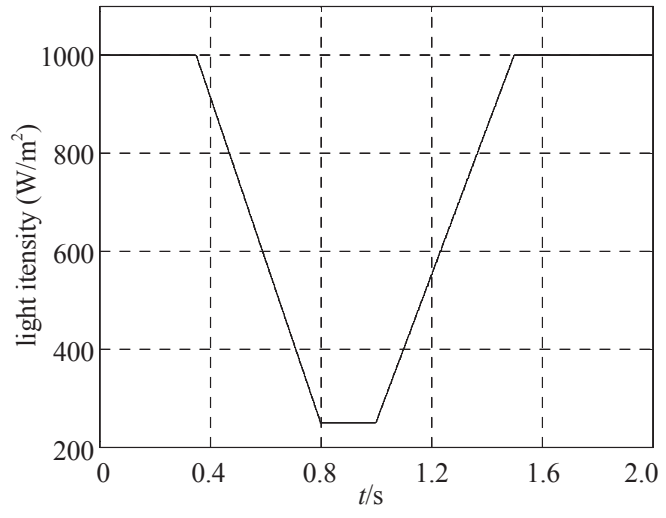

(a) Light intensity curve.

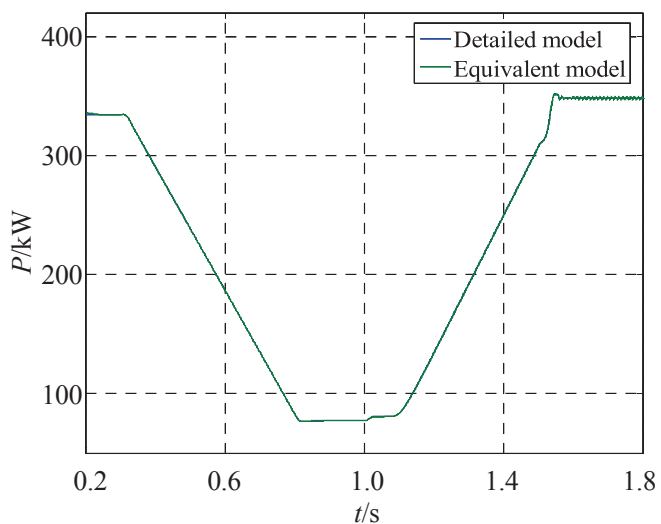

(b) Reactive power response curve.

Fig. 10. Response curves for light intensity disturbance.

ness of the proposed clustering modeling method again.

\section{4) Light Intensity Disturbance}

In this section, a comparison is shown when the external environment changes. In the simulation, the light intensity of the system is shown in Fig. 10(a). The active power response curves of the detailed model and the equivalent model at the PCC point are shown in Fig. 10(b).

As seen in Fig. 10, the duration of interruption in light intensity is long, and the system response is not severe. Therefore, by using maximum power point tracking (MPPT), the active power of the PV cluster can effectively track the change in light intensity and the active power response curves of the two models are very similar.

\section{CONCLUSION}

This paper presents an equivalent modeling method for regional decentralized PV clusters. By extracting feature points of response curves, the clustering index of a PV system is given. The PV systems are then divided into groups by a FCM clustering algorithm. PV systems that are in the same group are merged into an equivalent PV system by calculation of equivalent parameters, establishing an equivalent model of the regional decentralized PV cluster.

Simulation results and error analysis shows the effectiveness of the proposed method. The optimal grouping number is determined by the evaluation indices PC and CE, and the established equivalent PV system has a similar response to disturbances such as short-circuit faults, grid voltage fluctuation and external environment changes as the detailed model. Whether this method can be extended to other types of distributed generator cluster is a topic for further research.

\section{REFERENCES}

[1] M. Torres, J. Espinoza, and L. Moran et al., "Integration of a largescale photovoltaic plant using a multilevel converter topology and virtual synchronous generator control," in Proceedings International Conference on Symposium on Industrial Electronics, Istanbul, Turkey, Jun. 2014, pp. 2620-2624.

[2] D. Remon, A. M. Cantarellas, and M. A. A. Elsaharty et al., "Equivalent model of a synchronous PV power plant," in Proceedings International Conference on Energy Conversion Congress and Exposition, Montreal, Canada, Sep. 2015, pp. 47-53.

[3] D. Divan and P. Kandula, "Distributed power electronics: An enabler for the future grid," CPSS Transactions on Power Electronics and Applications, vol. 1, no. 1, pp. 57-65, 2016.

[4] D. Tan and D. Novosel, "Energy challenge, power electronics \& systems (PEAS) technology and grid modernization," CPSS Transactions on Power Electronics and Applications, vol. 2, no. 1, pp. 3-11, 2017.

[5] Y. Wang, X. Zhu, and Y. Wang, "Large-scale photovoltaic power station reliability model and energy-yield sensitivity analysis," in Proceedings International Conference on Electrical Machines and Systems, Pattaya, Thailand, Oct. 2015, pp. 1655-1659.

[6] H. M. Hasanien, "Shuffled frog leaping algorithm for photovoltaic model identification," IEEE Transactions on Sustainable Energy, vol 6, no. 2, pp. 509-515, 2015.

[7] S. Mukherjee, V. R. Chowdhury, and P. Shamsi et al., "Model reference adaptive control based estimation of equivalent resistance and reactance in grid-connected inverters," IEEE Transactions on Energy Conversion, vol. 32, no. 4, pp. 1407-1417, 2017.

[8] D. Remon, A. M. Cantarellas, and P. Rodriguez, "Equivalent model of large-scale synchronous photovoltaic power plants," IEEE Transactions on Industry Applications, vol. 52, no. 6, pp. 5029-5040, 2016.

[9] S. Li, Z. Liu, and X. Hao et al., "Dynamic equivalence to induction generators and wind turbines for power system stability analysis," in Proceedings International Conference on IEEE International Symposium on Power Electronics for Distributed Generation Systems, Hefei, China, Jun. 2010, pp. 887-892.

[10] A. J. Germond and R. Podmore, " Dynamic aggregation of generating unit models," IEEE Transactions on Power Apparatus and Systems, vol. 97 , no. 4, pp. 1060-1069, 1978.

[11] F. Ma and V. Vittal, "A hybrid dynamic equivalent using ANN-based boundary matching technique," IEEE Transactions on Power Systems, vol. 27, no. 3, pp. 1494-1502, 2012.

[12] H. B. Wu, S. Z. Wang, and B. Zhao et al., "Energy management and control strategy of a grid-connected PV/battery system," International Transactions on Electrical Energy Systems, vol. 25, no. 8, pp. 1590$1602,2014$.

[13] X. D. Cui, W. Li, and Z. W. Li et al., "An online dynamic equivalent method for large-scale photovoltaic power plant suitable for electromechanical transient stability simulation," Automation of Electric Power Systems, vol. 39, no. 12, pp. 21-26, 2015.

[14] N. Haghdadi, B. Asaei, and Z. Gandomkar, "Clustering-based optimal sizing and siting of photovoltaic power plant in distribution network," in Proceedings International Conference on IEEE International Conference on Environment and Electrical Engineering, Venice, Italy, May 2012, pp. 266-271.

[15] C. Li, J. Wang, and X. Li et al., "An optimized FCM method for electric load clustering," in Proceedings International Conference on Electric Utility Deregulation and Restructuring and Power Technologies, Nanjing, China, Apr. 2008, pp. 882-886.

[16] Y. B. Amlashi and H. Afrakhte, "Determination of wind plant output 
capacity using discrete Markov chains and PSO methods in comparison with FCM," International Review on Modelling \& Simulations, vol. 4, no. 2, pp. 819-823, 2011.

[17] J. Zou, C. Peng, and H. Xu et al., "A fuzzy clustering algorithm-based dynamic equivalent modeling method for wind farm with DFIG," IEEE Transactions on Energy Conversion, vol. 30, no. 4, pp. 1329$1337,2015$.

[18] L. L. Huang, N. Chen, and H. Zhang et al., "Optimization of largescale offshore wind farm electrical collection systems based on improved FCM," in Proceedings International Conference on Sustainable Power Generation and Supply, Hangzhou, China, Sep. 2012, pp. 1-6.

[19] X. P. Pan, Y. Zhang, and P. Ju et al., "Equivalent modeling for photovoltaic power station," Power System Technology, vol. 39, no. 5, pp. 1173-1178, 2015.

[20] Z. Ma, J. Zheng, and S. Zhu et al., "Online clustering modeling of large-scale photovoltaic power plants," in Proceedings International Conference on IEEE Power \& Energy Society General Meeting, Colorado, America, Jul. 2015, pp. 1-5.

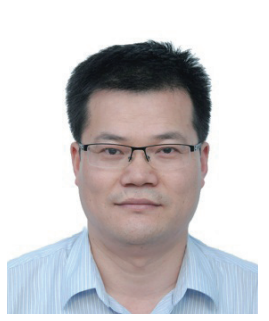

Hongbin Wu received his B.Sc., M.Sc. and Ph.D. degrees in Electrical Engineering from Hefei University of Technology, Hefei, China in 1994, 1998 and 2005 respectively. He is now a professor of Hefei University of Technology. His research field includes distributed generation technology and distribution network modeling and simulation.

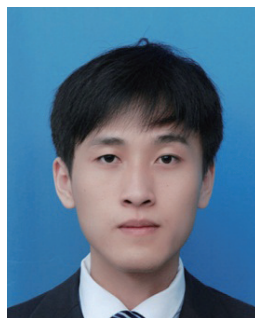

Zhongqian Liu received his B.S. degrees in electrical engineering and automation from Hefei University of Technology in 2015. He is now purchasing his M.S. degrees in power system and automation from Hefei University of Technology. His research interest includes distributed power generation technology.

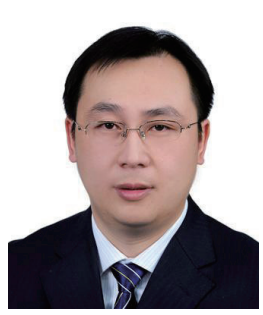

Yu Chen was born in 1976. He received his M.S. degrees in electrical engineering and automation from Shanghai Jiao Tong University in 2016. He is now a senior engineer of State Grid Anhui Economic Research Institute. His research areas include electric economical and distribution network system planning.

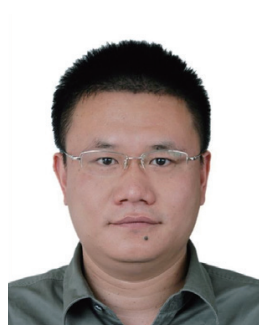

Bin Xu was born in 1970. He received his M.S. degrees in electrical engineering and automation from Hohai University in 2000. He is now a senior engineer of State Grid Anhui Electric Power Research Institute. His research areas include distribution network system planning and design techniques.

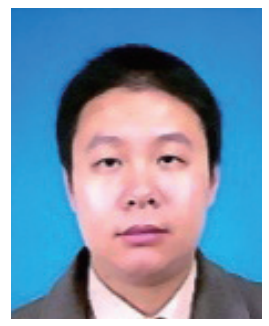

Xianjun Qi was born in 1977. He received his B.Sc. M.Sc. and Ph.D. degrees in Electrical Engineering from Hefei University of Technology, Hefei, China in 2000, 2003 and 2009 respectively. He is now an associate professor of Hefei University of Technology. His research field includes distributed generation technology and distribution network system analysis and planning. 